\title{
Carbon Nanostructures Synthesized by Chemical Reaction Using Rongalite and Polyethyleneimine as Complex Agents
}

\author{
J.A. González ${ }^{1}$, R. C. Carrillo-Torres ${ }^{1}$, M. E. Alvarez-Ramos ${ }^{1}$ and S. J. Castillo ${ }^{2}$ \\ 1. Departamento de Física, Universidad de Sonora, Hermosillo, Sonora, México. \\ 2. Departamento de Investigación en Física, Universidad de Sonora, Hermosillo, Sonora, México.
}

Carbon atoms form various allotropes with different physical and chemical natures originated from the variety of $s-p$ orbital hybridization $[1,2]$. Carbon is a remarkable element showing a variety of stable forms ranging from 3D semiconducting diamond to 2D semi-metallic graphite to ID conducting and semiconducting carbon nanotubes to OD fullerenes, in this work we present a synthesis route to carbon with hierarchical morphology on the nanoscale.

Carbon nanostructures have been synthetized by chemical reaction in aqueous solution using saccharose, deionized water, rongalite and sulfuric acid as precursor reagents. The main idea is to carbonize the saccharose by means of a dehydrolization process with sulfuric acid. Rongalite was used to complex some intermediate carbon structures due its powerful reductor capability.

1gr of saccharose was mixed with $2 \mathrm{ml}$ of deionized water in a glass beaker at room temperature until it dissolved, then $0.3 \mathrm{ml}$ of polyethyleneimine (PEI, $\left.\mathrm{C}_{2} \mathrm{H}_{5} \mathrm{~N}_{2}\right), 0.3 \mathrm{ml}$ of rongalite $\left(\mathrm{CH}_{3} \mathrm{NaO}_{3} \mathrm{~S}\right), 3 \mathrm{ml}$ of sulfuric acid $\left(\mathrm{H}_{2} \mathrm{SO}_{4}\right)$ and $0.3 \mathrm{ml}$ of ammonium hydroxide $\left(\mathrm{NH}_{4} \mathrm{OH}\right)$ buffer $\mathrm{pH} 11$ was added to the mixture. At first the solution become darker and further addition of sulfuric acid made the solution completely black.

The material obtained was characterized by FTIR and Raman spectroscopy and SEM-EDS studies.

The samples where characterized by infrared transmission spectra carried out by a Horiba- Jobin-Yvon LabRam HR with a He-Ne laser at $632.8 \mathrm{~nm}$, the chemical reaction give rise to a peak at $1645 \mathrm{~cm}^{-1}$, which is corresponding to a stretching vibration mode of $\mathrm{C}=\mathrm{C}$ bond, the intensities, positions and widths of the observed peaks are well consistent for the most part in the spectrum, it is inferred that the $\mathrm{C}=\mathrm{C}$ bond was formed by a dehydration of the saccharose.

The Raman spectra of the sample are show in figure 2, it was carried out by a Perkin Elmer Spectrum two with a UATR module, the spectrum was normalized by the maximum values of G-band peak, the spectrum was collected in the range from 0 to $4000 \mathrm{~cm}^{-1}$, the peaks shown are the so called "G-peak" (for graphite) at $1584 \mathrm{~cm}^{-1}$ and "D-peak" (for disorder) at $1358 \mathrm{~cm}^{-1}$. Band peaks characteristics are strongly dependent on structure of the graphitization [3] and the Raman spectra are interpreted by models and theories [4]. The G-peak is due to the phonon mode that allowed by a break-up of selection rules for crystallite with sizes smaller than 100nm (nanocrystalline graphite), the spectra also shows a strong peak in the $2500 \mathrm{~cm}^{-1}$ characteristic of the $\mathrm{sp}^{2}$ carbon materials.

The scanning electron microscopy (SEM) were carried out by a FE-SEM JOEL jsm-7800F, images of the formed particles are shown in the figures 3 and 4, the sample has a ordained and homogenous morphology, the surface is covered by small nano-sized grains of about $100 \mathrm{~nm}$. 
The Elemental composition of the samples has been determined from EDS. The EDS spectrum show the presence of $\mathrm{C}$ of $41.88 \%$ and $47.36 \%$ of oxygen in atomic mass percentages, also a mapping EDS analysis shows a homogenous elemental composition of carbon, oxygen, sulfur, sodium and nitrogen in the sample [5].

\section{References:}

[1] B. McEnaney, in: T.D. Burchell (Ed.), Carbon Materials for Advanced Technologies, (Pergamon, Oxford) 1999 (Chapter 1).

[2] M. Inagaki, L.R. Radovic, Nanocarbons, Carbon 40 (2002), p. 2279.

[3] P. Lespade, R. Al-Jishi, M.S. Dresselhaus, Carbon 20 (1982), p. 427.

[4] A.C. Ferrari, J. Robertson, Phys. Rev. B 61 (2000), p. 14095.

[5] The authors acknowledge to CONACyT with project number 255791-INFR-2015.

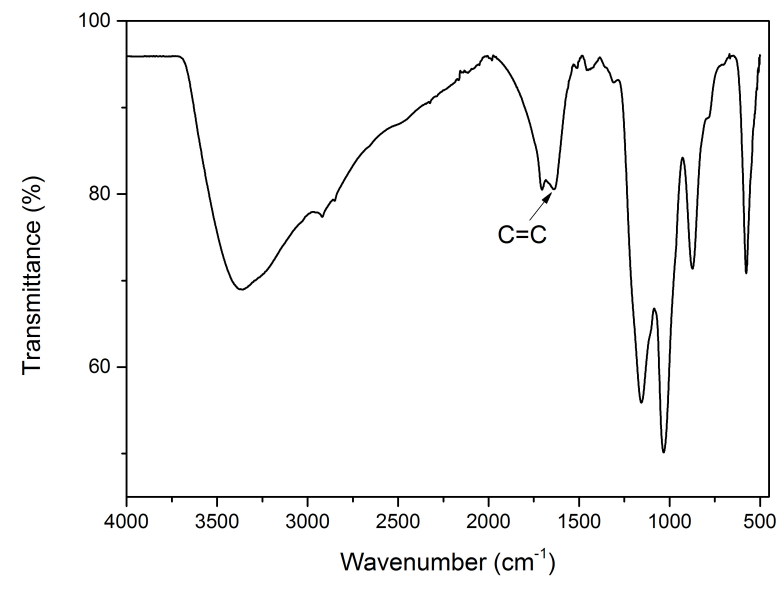

Figure 1. Infrared spectra of the nanostructure carbon Samples.

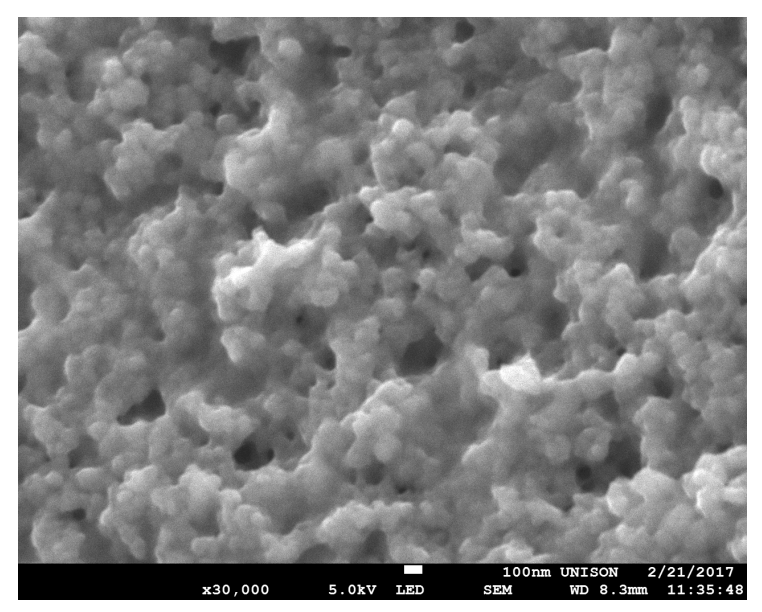

Figure 3. SEM image of the sample with 30000 magnification.

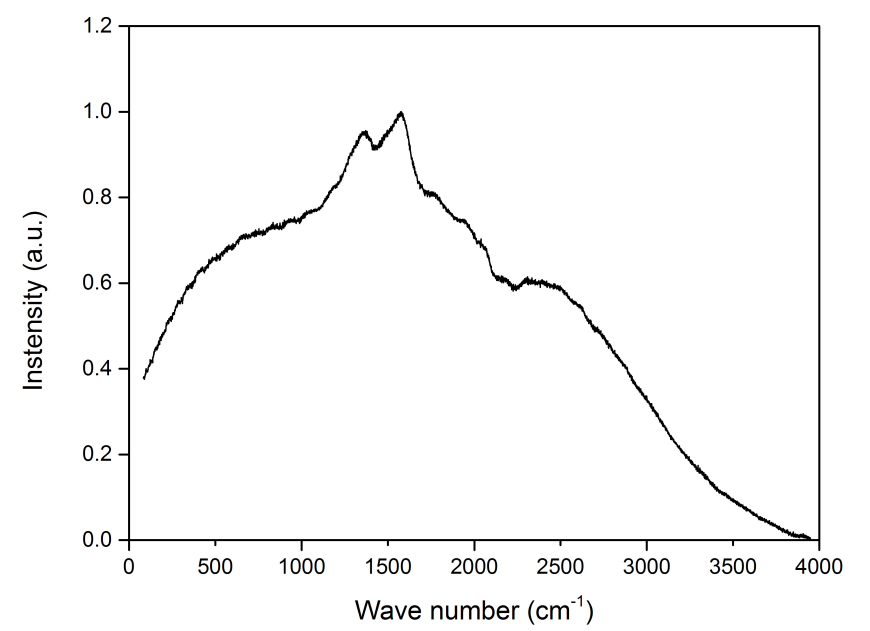

Figure 2. Raman spectra of the samples

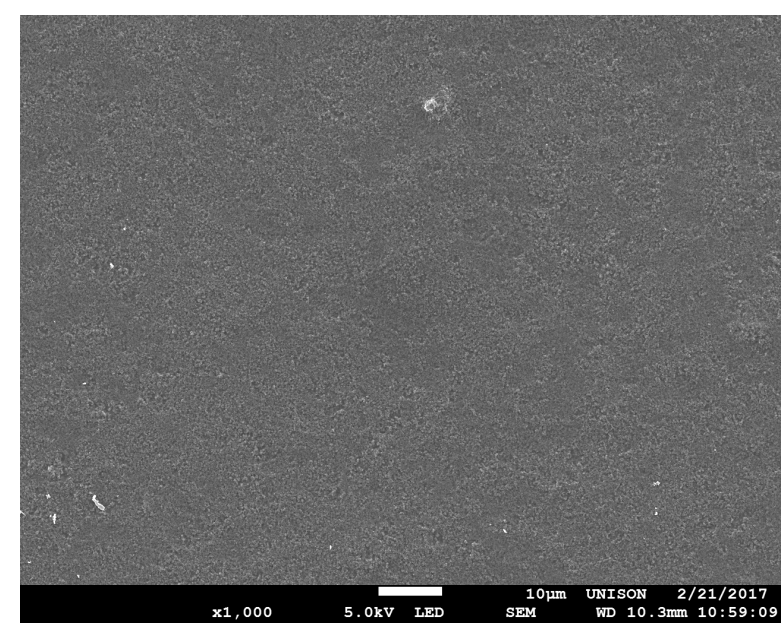

Figure 4. SEM image of the sample with 15000 magnification. 